\title{
LCA robustly reveals subtle diversity in large-scale single-cell
}

RNA-seq data

3

4 Changde Cheng ${ }^{1}$, John Easton ${ }^{1}$, Celeste Rosencrance ${ }^{1}$, Yan Li ${ }^{2}$, Bensheng Ju ${ }^{1}$, Justin

5 Williams ${ }^{1}$, Heather L Mulder ${ }^{1}$, Wenan Chen $^{1} \&$ Xiang Chen*1

7 Author affiliation:

$8{ }^{1}$ Department of Computational Biology, St. Jude Children's Research Hospital,

9 Memphis, Tennessee 38105, USA

10 2Division of Biostatistics, University of Minnesota, Minneapolis, Minnesota 55455, USA

13 *Correspondence should be addressed to Xiang Chen (xiang.chen@stjude.org). 


\section{Abstract}

16 Single-cell RNA sequencing has emerged as a powerful tool for characterizing the cell-

17 to-cell variation and dynamics. We present Latent Cellular Analysis (LCA), a machine

18 learning-based analytical pipeline that features a dual-space model search with

19 inference of latent cellular states, control of technical variations, cosine similarity

20 measurement, and spectral clustering. LCA has proved to be robust, accurate, scalable,

21 and powerful in revealing subtle diversity in cell populations.

22

\section{Keywords}

24 Clustering, Single-cell RNA-seq 


\section{Backgrounds}

26 Single-cell RNA sequencing (scRNA-seq) enables the characterization of cell-to-cell

27 variation in transcript abundance, leading to a deep understanding of the diversity of cell

28 types and the dynamics of cell states in populations of thousands to tens of thousands

29 of single cells [1-3]. Although scRNA-seq offers enormous opportunities and has

30 inspired a tremendous explosion of data-analysis methods for identifying heterogeneous

31 subpopulations, significant challenges can arise because of the inherently high noise

32 associated with data sparsity and the ever-increasing number of cells sequenced. The

33 current state-of-the-art algorithms have significant limitations. The biological meaning of

34 the cell-to-cell similarity learned by most machine learning-based tools (such as Seurat

35 [4], Monocle2 [5], SIMLR [6], and SC3 [7]) is difficult to interpret. Several methods

36 require the user to provide an estimation of the number of clusters in the data, and this

37 may not be readily available. Furthermore, most methods have a high computational

38 cost that will be prohibitive for datasets representing large numbers of cells. Lastly,

39 although certain technical biases (e.g., cell-specific library complexity) have been

40 recognized as major confounding factors in scRNA-seq analyses [8], other technical

41 variations (e.g., batch effects and systematic technical variations that are irrelevant to

42 the biological hypothesis being evaluated) have received minimal attention, even though

43 they present major challenges to the analyses [9]. Most methods employ a gene-

44 selection step before clustering analysis, based on the assumption that a small subset

45 of highly variable genes is most informative for revealing cellular diversity. Although this

46 assumption may be valid in many scenarios, it fails when informative genes are not 
47 most variable. This can happen when the difference among subpopulations is subtle, or

48 there is a strong batch effect, while most variable genes differ by batch.

50 To address these issues, we developed Latent Cellular Analysis (LCA), an accurate,

51 robust, and scalable computational pipeline that will facilitate a deep understanding of

52 the transcriptomic states and dynamics of single cells in large-scale scRNA-seq

53 datasets. LCA makes a robust inference of the number of populations directly from the

54 data (a user can specify this with a priori information), rigorously models the

55 contributions from potentially confounding factors, generates a biologically interpretable

56 characterization of the cellular states, and better reveals the underlying population

57 structures. Furthermore, LCA addresses the scalability problem by learning a model

58 from a subset of the sample, after which a theoretical scheme is used to assign the

59 remaining cells to identified populations.

60

\section{Results}

\section{Overview of the LCA method}

63 LCA employs a heuristic dual-space search approach (Fig. 1) to provide an optimal

64 model for subpopulation structure identification (including the removal of confounding

65 factors, the inference of the number of clusters and informative states, and a mapping

66 function from the expression vector to cluster membership). In the primary latent cellular

67 (LC) space, LCA bypasses gene selection and performs LC state inference from the

68 global gene expression matrix, removes cellular states representing known confounding

69 factors (e.g., cell-specific library complexity and batch information), and measures cell- 
70 to-cell similarity by using the cosine of the angle between the low-dimensional cellular-

71 state vectors. Spectral clustering is employed to derive a set of candidate clustering

72 models with a range of cluster numbers. Meanwhile, in the dual principle component

73 (PC) space, cells are projected into a low-dimensional space based on principal

74 component analysis (PCA) of the expression vector. The list of significant components

75 is determined by the Tracy-Widom test [10], and cell-to-cell similarity is measured by

76 correlation similarity in the PC space. LCA then ranks candidate clustering models

77 derived in the LC space by the silhouette index [11] measured in the PC space. Finally,

78 LCA retains informative cellular states from the selected clustering model(s) and uses

79 these states to update the final clustering solution(s). Although the "optimal model" is

80 not necessarily the solution with the best numerical score, it should be among the top-

81 ranked models. Therefore, LCA provides users with multiple top-ranked solutions for

82 biology-based evaluations.

\section{LCA accurately identifies cellular states in simulated datasets}

85 We benchmarked LCA with four commonly used state-of-the-art scRNA-seq clustering

86 algorithms (SC3 [7], Seurat [4], Monocle2 [5], and SIMLR [6]) in 3000 simulated

87 datasets. LCA outperformed competing methods in subpopulation number inference,

88 cluster membership inference, and running time.

90 We simulated three equally spaced cell subpopulations of various sizes by shuffling a

91 predetermined number of genes across cells from high-quality, Unique Molecular

92 Identifier (UMI)-based scRNA-seq data. We simulated seven scenarios with increasing 
93 signal strength, varying the number of simulated differentially expressed genes (DEGs),

94 the fold change among groups, and the average expression levels of the simulated

95 DEGs. We also investigated the effect of the sample size (from 250 to 4000 cells) on

96 the performance of the algorithms.

98 A typical clustering analysis partitions data points into meaningful subgroups without a

99 priori knowledge of the number of subsets or their compositions. An essential

100 component of clustering analysis is inferring the number of clusters from the data [12],

101 which presents an analytical challenge distinct from that posed by clustering with a

102 given number of clusters. Therefore, we first analyzed the accuracy and robustness of

103 the subpopulation number inference. Monocle2 and SIMLR were excluded from this

104 analysis because both algorithms require an input of the number of clusters from end-

105 users. Fig. 2A shows that although LCA started with a slightly off-target estimation of

106 the cluster number in datasets with very weak signal strength, it stably converged to the

107 correct number with increasing signal strength from the number of cells, the number of

108 DEGs, the fold difference, or the expression level. In contrast, using SC3 or Seurat

109 resulted in an upward bias in the estimation of the number of clusters, and this bias

110 increased with both the sample size and the signal strength. For example, in simulated

111 model \#6, with the strongest signal strength, the actual cluster number remained the

112 same (three) for all sample sizes, but SC3 estimated medians of four, eight, and sixteen

113 clusters in datasets with 250, 1000, and 4000 cells, respectively. Seurat estimated

114 medians of three, four, and nine subpopulations in the same respective datasets.

115 Combining the results of all simulations, we find that LCA arrived at the correct number 
116 of clusters in $70.2 \%$ of simulations, which is a significantly higher percentage than was

117 achieved with SC3 $\left(5.6 \%, P<2.2 \times 10^{-16}\right.$ [proportion test]) and Seurat $(22.7 \%$,

$118 P<2.2 \times 10^{-16}$ [proportion test]).

120 We used the variation of information (VI [13], 0 for perfect matching) (Fig. 2B) and

121 normalized mutual information (NMI [14], 1 for perfect matching) (Fig. 2C) to measure

122 the clustering accuracy. For methods with a feature for inferring the number of clusters

123 from the data (LCA, SC3, and Seurat), we compared the ground-truth labels with

124 inferred cluster membership from the optimal clustering models. For methods without

125 this inference feature (Monocle2 and SIMLR), the optimal models were derived with the

126 correct number of clusters (three). The comparison showed that LCA outperformed

127 other methods in most simulations (Fig. 2). When all 3000 simulation datasets were

128 combined, LCA achieved the highest overall accuracy (average NMI = 0.756;

$129 \mathrm{VI}=0.555)$. Specifically, when LCA correctly inferred the number of clusters (in $70.2 \%$

130 of all simulations), it produced near-perfect accuracy (average $\mathrm{NMI}=0.941, \mathrm{VI}=0.122$ ).

131 The accuracies of the competing algorithms were consistently lower. Notably, SC3

132 achieved a relatively good performance $(\mathrm{NMI}=0.807, \mathrm{VI}=0.389)$ when it happened to

133 find the correct number of clusters (mostly in small datasets). However, its performance

134 deteriorated for other datasets, primarily due to an overestimation of cluster numbers.

135 The overall accuracy of SC3 was significantly lower than that of LCA $(\mathrm{NMI}=0.583$,

$136 P<2.2 \times 10^{-16} ; \mathrm{VI}=1.203, P<2.2 \times 10^{-16}$ [Wilcoxon signed-rank test]). More strikingly,

137 even if provided with the correct cluster number, Monocle2 attained high accuracy only

138 for the dataset with the strongest signal strength (simulated model \#6), and its 
139 performance was suboptimal compared to that of LCA (NMI $=0.190, P<2.2 \times 10^{-16}$; $\mathrm{VI}=1.719, P<2.2 \times 10^{-16}[$ Wilcoxon signed-rank test]). Similarly, the overall accuracies

141 of Seurat $\left(\mathrm{NMI}=0.415, P<2.2 \times 10^{-16} ; \mathrm{VI}=1.545, P<2.2 \times 10^{-16}\right.$ [Wilcoxon signed-rank

142 test]) and SIMLR (NMI $=0.231, P<2.2 \times 10^{-16} ; \mathrm{VI}=1.353, P<2.2 \times 10^{-16}[$ Wilcoxon

143 signed-rank test]) were significantly lower than that of LCA.

145 Scalability to large data sets

146 In addition to the superior accuracy of LCA with respect to both cluster number and

147 membership assignment, its implementation renders exceptional scalability, which is an

148 attractive feature for scRNA-seq analysis of an ever-increasing number of cells. LCA

149 can derive a cell subpopulation structure by using a relatively small representative set

150 (training cells) sampled from the full data. LCA provides mathematical formulae with

151 which to project the remaining cells (testing cells) directly to the inferred low-

152 dimensional LC space, after which individual cells are assigned to the subpopulation

153 with the best similarity. Consequently, LCA runs at a low level of computational

154 complexity. The median overall running times for a 4000 -cell dataset with $10 \%$ or $25 \%$

155 training cells were $40.6 \mathrm{~s}$ and 202.9 s, respectively. We compared the accuracy of

156 clustering between the training cells (5\%-25\% of all cells) and the remaining testing

157 cells in two simulated models (Fig. 2C, D, models \#2 and \#6). As expected, the

158 accuracy of the clustering model improved with the increase of training cells in the

159 simulated dataset \#2 (weak signal strength) and remained at a near-perfect level in

160 simulated dataset \#6 (strong signal strength). Strikingly, LCA achieved comparable 
161 accuracies in the testing cells in both models and outperformed SC3 in terms of

162 accuracy and running time.

\section{Evaluation of LCA on published data sets}

165 By using publicly available large-scale scRNA-seq datasets, we demonstrated that LCA

166 identified orthogonal LC states of biological importance and produced parsimonious and 167 accurate models that were highly consistent with biological knowledge.

169 We evaluated the performance of LCA with large-scale datasets published by Zheng et

170 al. [15] and Tirosh et al. [16]. Using the GemCode platform (10x Genomics, Pleasanton,

171 CA), Zheng et al. generated reference scRNA-seq transcriptomes for subpopulations of

172 peripheral blood mononuclear cells (PBMCs) purified via well-established cell surface

173 markers [15]. We generated a large-scale purified T-cell dataset (representing 55,000

174 cells) by combining the CD4+ T-helper (CD4+ helper), CD4+/CD25+ regulatory $\mathrm{T}\left(\mathrm{T}_{\text {reg }}\right)$,

175 CD4+/CD45RO+ memory $T\left(T_{\text {mem }}\right)$, CD4+/CD45RA+/CD25- naïve T (CD4+ ab T),

176 CD8+ cytotoxic T (cytotoxic T), and CD8+/CD45RA+ naïve cytotoxic T (CD8+ ab T) cell

177 subpopulations. We inferred the cellular states and subpopulation structure by using

$17810 \%$ and $25 \%$ of the full dataset, and we assigned the remaining cells to one of the

179 inferred subpopulations. We evaluated the top three models for both runs, which were

180 the 3-population, 4-population, and 5-population models. Despite a 9.6-fold difference in

181 running time, both the 3-population and 5-population models from the two runs achieved

182 high consistency ( $\mathrm{NMI}=0.87$ and 0.84 , respectively). Although the 4-population models

183 differed between runs, they represented two different subpopulation-merging orders 
184 from the 5-population model to the 3-population model. We selected the 5-population

185 model learned from $25 \%$ cells (with 19 LC states) for further biological inference.

186 Whereas the purified $\mathrm{T}_{\mathrm{mem}}, \mathrm{CD} 4+\mathrm{ab} \mathrm{T}$, and CD8+ ab T cells contained cells mostly from

187 a single subpopulation (Clusters 1, 3, and 4, respectively), different levels of

188 heterogeneity were detected in the remaining purified populations, especially the CD4+

189 helper and cytotoxic T cells. Given the single surface-marker settings in the purification

190 of CD4+ helper and cytotoxic T cells, it is not surprising that we found substantial

191 heterogeneity in the two populations. Nevertheless, LCA inferred a parsimonious

192 subpopulation structure in this large dataset (compared to the 1108 subpopulations

193 predicted by SC3). We selected representative genes encoding surface markers,

194 transcription factors, and secreted effector molecules for 19 usual T-cell subsets [17]

195 and derived a PCA projection of the six purified populations and five inferred clusters

196 based on the average population/cluster expression level of individual marker genes.

197 The first PC largely described the differentiation status, and the second PC represented

198 the difference between the CD4+ subsets and CD8+ subsets. As expected, Clusters 3

199 and 4 were found next to the CD8+ ab T and CD4+ ab T cells. Cluster 1 was found near

200 the $\mathrm{T}_{\text {mem }}$ population. Cluster 2 consisted mostly of $\mathrm{T}_{\text {reg }}$ cells and a smaller fraction of

201 CD4+ helper cells that was located adjacent to the $T_{\text {reg }}$ population. Both the CD4+

202 helper and cytotoxic T-cell populations were split approximately equally between naïve

203 and differentiated cells and were spotted between their corresponding naïve clusters

204 (Cluster 3 for CD8+ cells and Cluster 4 for CD4+ cells) and differentiated clusters

205 (Clusters 1 and 2 for CD4+ cells and Cluster 5 for CD8+ cells). Analysis of selected

206 genes validated the separation of naïve and differentiated cells in the CD4+ helper and 
207 cytotoxic T cells (Fig. 3). An evaluation of the first LC state inferred from the full

208 expression data revealed a striking approximation to the expected distribution of naïve

209 and differentiated cells in both purified populations and inferred clusters. Similarly, the

210 second LC state recaptured the CD4+ and CD8+ difference in the dataset. These

211 results showed that LCA identified LC states of biological importance and consequently

212 produced a parsimonious and accurate model that was highly consistent with biological

213 knowledge.

214

215 We evaluated the performance of LCA in a second dataset published by Tirosh et al.

216 [16], who employed a stepwise approach to analyze separately the malignant and

217 stromal cells of 19 melanoma tumors captured on the C1 Fluidigm platform. We applied

218 those authors' cell-selection criteria [16] to generate a dataset with 1169 malignant cells

219 from eight tumors and 2588 nonmalignant (stromal) cells. LCA inferred 18 clusters with

22053 LC states (Fig. 4). Malignant cells dominated eight clusters, which were further

221 separated by the patient origin of the tumors (Fig. 4A). Among the stromal cells, distinct

222 clusters were identified for B cells, macrophages, cancer-associated fibroblasts, and

223 endothelial cells (Fig. 4B). Moreover, LCA divided tumor-infiltrating T cells and natural

224 killer cells into six clusters, which was concordant with the supervised analysis of T cells

225 based on surface markers by Tirosh et al. [16]. Using the pre-defined marker genes for

226 T-cell subsets and MKI67 for cell-cycle activities, we revealed characteristics of these T-

227 cell populations (Fig. 4C). Cluster 1 was enriched for naïve CD4+ cells, whereas Cluster

2282 harbored mostly $\mathrm{T}_{\text {reg }}$ and $\mathrm{T}_{\mathrm{FH}}$ cells. Although Clusters 3 and 5 both had enriched

229 signatures for cytotoxic T and exhaustive T cells, Cluster 3 had greater signal strength 
230 in both signatures, consistent with the reported high correlation between exhaustion

231 markers and cytotoxic markers [16]. Cells in active cell cycles were grouped in Cluster

232 4, which showed unique enrichment of MKI67 expression. Lastly, natural killer cells and

233 T cells with weak cytotoxic activity and no exhaustion signatures were found in Cluster

234 6. As a comparison, we applied SC3 to the same data, which resulted in an estimation

235 of 43 clusters. Although SC3 clustering of malignant cells was consistent with the

236 separation by patient, accuracy in the stromal component was lower than with LCA

237 (Supplementary Table 1). These results demonstrate the power of LCA to reveal

238 subtle diversities in different subpopulations of tumor-infiltrating $\mathrm{T}$ cells in the presence

239 of strong transcriptomic variations among malignant cells (from different patients) and

240 various stromal cells from different lineages (cancer-associated fibroblasts,

241 macrophages, B cells, and endothelial cells).

243 LCA work robustly on a data set with strong batch effects

244 LCA groups cells based on orthogonal LC states aligned with major differences among

245 cells, which enables control of technical variations (e.g., batch effects) without an

246 explicit need of gene filtering. We evaluated the efficiency of batch effect removal in a

247 cancer cell dataset and further experimentally validated the subpopulations revealed by

248 LCA. Rhabdomyosarcoma is the most common soft-tissue tumor in children and has

249 two major histologic subtypes with different genomic landscapes: PAX3/PAX7-FOXO1

250 fusion-positive alveolar rhabdomyosarcoma (FP-ARMS) and fusion-negative embryonal

251 rhabdomyosarcoma (ERMS) [18]. LCA identified two subpopulations in a scRNA-seq

252 dataset for Rh41 cells (a commonly used human PAX3-FOXO1 FP-ARMS cell line). 
253 The CD44 gene, which encodes a commonly used cell surface marker with great

254 prognostic and therapeutic potential [19, 20], appeared at the top of the differentially

255 expressed genes (DEGs) of the two subpopulations (Supplementary Figure 1A). Flow

256 cytometry confirmed a bimodal expression pattern of CD44 in Rh41 cells

257 (Supplementary Fig. 1B). We first used bulk RNA-seq to profile unsorted populations

258 and CD44 $4^{\text {high }}$ and CD44 ${ }^{\text {low }}$ subpopulations sorted by fluorescence-activated cell sorting.

259 In addition to the differences among the sorted CD44high, CD44 ${ }^{\text {low }}$, and unsorted

260 populations, the analysis revealed strong batch effects. Specifically, samples in Batch 1

261 and those in Batches 2 and 3 were separated on the first PC, whereas the biologically

262 different populations (the unsorted population and sorted subpopulations) were

263 separated on the second PC (Supplementary Fig. 1C). We collected scRNA-seq data

264 for the unsorted populations in Batch 1 and the sorted CD44 $4^{\text {high }}$ and CD44low

265 subpopulations in Batch 2 (10x Genomics, Pleasanton, CA). The libraries were

266 sequenced at different depths for the three samples. The median numbers of UMIs

267 captured per cell were 8278,6678 , and 12,850 for the CD44 ${ }^{\text {high }}$ subpopulation, CD44 ${ }^{\text {low }}$

268 subpopulation, and unsorted population, respectively (Kruskal-Wallis rank sum test,

$\left.269 P<2.2 \times 10^{-308}\right)$. With both batch effects and biological difference among cell

270 populations, LCA inferred five clusters, with cells in Batch 1 being represented by

271 Clusters 1 and 2 and cells in Batch 2 being grouped in Clusters 3 to 5 (Supplementary

272 Fig. 1D). Similarly, SC3 inferred a 12-cluster structure. Consistent with the strong batch

273 effects observed in bulk RNA-seq analysis, when required to infer a two-cluster model,

274 both LCA and SC3 achieved a perfect separation based on the batch information

$275(\mathrm{NMI}=1$ and 0.99, respectively). Next, we evaluated the association between the batch 
276 covariate and individual LC states. When controlled at FDR $\leq 0.05$, the first LC state was

277 the only one significantly associated with the batch information $\left(P<2.2 \times 10^{-308}\right)$

278 (Supplementary Fig. 1E). After excluding this state, LCA retrieved an optimal structure

279 with two clusters. Of 7005 cells profiled in the sorted CD44 ${ }^{\text {low }}$ subpopulation, 6733

$280(96.1 \%)$ were clustered into Cluster 2, suggesting that the subpopulation was relatively

281 pure. However, 1796 (26.6\%) of 6757 of cells profiled in the sorted CD44high

282 subpopulation were grouped in Cluster 2 with the CD44 ${ }^{\text {low }}$ cells (Fig. 5A). We evaluated

283 the expression signatures for DEGs identified in bulk RNA-seq $\left(\log _{2} F C \geq 1, F D R \leq 0.05\right.$,

284 FPKM $\geq 1$ in at least one subpopulation). Of 1709 DEGs (Supplementary Table 2), 356

285 were captured in at least $10 \%$ of the cells in one sequenced population or inferred

286 cluster. As expected, Clusters 1 and 2 had higher average expression levels of genes

287 overexpressed in the bulk-sorted CD44 $4^{\text {high }}$ and CD44 low subpopulations, respectively

288 (Fig. 5B). Importantly, Cluster 2 cells from the sorted CD44 ${ }^{\text {high }}$ subpopulation had

289 significantly lower expression of those DEGs overexpressed in the bulk CD44high

290 subpopulation than did Cluster 1 cells from the sorted CD44 ${ }^{\text {high }}$ subpopulation

$291\left(P<2.2 \times 10^{-308}\right)$ or the sorted CD44 ${ }^{\text {low }}$ subpopulation $\left(P=7.5 \times 10^{-71}\right)$. Analysis of DEGs

292 overexpressed in the bulk CD44 ${ }^{\text {low }}$ subpopulation showed the same pattern (Fig. 5C).

293 These results suggested that Cluster 2 cells in the sorted CD44 ${ }^{\text {high }}$ subpopulation more

294 closely resembled CD44 ${ }^{\text {low }}$ cells. Moreover, the differential expression pattern was

295 essentially captured by the first remaining LC state (Spearman correlation

296 coefficient $\left.=-0.90, P<2.2 \times 10^{-308}\right)$, confirming the biological importance of the inferred

297 LC states. 
299 Of the three established molecular markers (TFAP2B, MYOG, and NOS1) for FP.

300 ARMS[21], TFAP2B and MYOG were overexpressed in the sorted CD44 low

301 subpopulation (Supplementary Table 2). Moreover, known PAX3-FOXO1 target genes

302 were significantly enriched in DEGs overexpressed in the CD44 ${ }^{\text {low }}$ subpopulation

303 (Supplementary Table 3). These results suggest that the CD44 ${ }^{\text {high }}$ subpopulation

304 represents a less-differentiated, stem-like cell subpopulation. Although the exact

305 mechanism by which the distinct subpopulations develop warrants further investigation,

306 we conclude that LCA can control technical variations and reveal reliable transcriptome-

307 based heterogeneity.

308

\section{Discussion and conclusions}

310 The rapid technological advance in scRNA-seq platforms has inspired a tremendous

311 explosion of data-analysis methods for identifying heterogeneous subpopulations. Most

312 methods employ a gene-selection step before clustering analysis, based on the

313 assumption that a small subset of highly variable genes is useful for revealing cellular

314 diversity. Although this assumption is valid in most scenarios and reduces the data

315 dimensionality, it potentially excludes genes that are informative for separating

316 subpopulations with subtle diversity. Also, in datasets with strong batch effects, it may

317 result in a small set of retained genes being dominated by batch effects. Moreover,

318 several methods require the user to provide an estimation of the number of clusters in

319 the data, and this may not be readily available. 
321 Using a dual-space search strategy, LCA bypasses the gene selection, learns

322 biologically informative cellular states directly from the raw gene expression matrix,

323 reduces potential technical variations, and measures the cell-to-cell distance by using

324 cosine similarity in the low-dimensional and informative cellular-state space in a data-

325 driven and unsupervised fashion. Cosine similarity has been widely used in information

326 retrieval and text mining to reveal the relation between text documents, a process that

327 shares many similarities with scRNA-seq analysis [22]. Furthermore, LCA provides a

328 mathematical solution for assigning new cells to inferred clusters in a model learned

329 from a subset of cells, a capability that is urgently needed to handle the ever-increasing

330 sample sizes in scRNA-seq. We have demonstrated through extensive simulation and

331 the use of large-scale scRNA-seq datasets that LCA is an efficient, scalable, and robust

332 clustering algorithm that outperforms other tools without the explicit need for gene

333 selection or an estimation of the number of clusters in the data.

\section{Methods}

\section{Latent cellular states}

337 The input to LCA is a gene expression matrix in which each column is a cell, and each

338 row is a gene/transcript. In UMI-based platforms, the expression level of a gene in a cell

339 is divided by the total expression in that cell to generate a relative expression matrix (T).

340 In read-count based platforms, T can be derived from size factor normalized expression

341 measures. The relative expression matrix is then log-transformed after adding a zero-

342 correction term:

$$
X=\log (T+\epsilon)
$$


344 where $\epsilon$ is an arbitrarily small number.

345

346 We obtain the LC states from a singular value decomposition (SVD) of $\mathbf{X}$.

$$
\mathbf{X}=\mathbf{G} \boldsymbol{\Lambda} \mathbf{S}^{T}
$$

348 where $\mathbf{S}$ is a cell-by-LC states matrix. We note that under certain conditions, $\mathbf{S}$ is the

349 same as the loading matrix from the principle component analysis (PCA) result of $\mathbf{X}$ [23,

$35024]$

\section{Determination of significant LC states}

352 We apply the Tracy-Widom test to associated eigenvalues to determine which LC states

353 are significant $[10,25,26]$. The LC state associated with the eigenvalue $\lambda$ is significant if

354 it is significantly different $(P<0.05)$ from the Tracy-Widom distribution, with

355

$$
\begin{aligned}
& \mu(n, m)=\frac{(\sqrt{m-1}+\sqrt{n})^{2}}{m} \\
& \sigma(n, m)=\frac{(\sqrt{m-1}+\sqrt{n})}{m}\left(\frac{1}{\sqrt{m-1}+\sqrt{n}}\right)^{\frac{1}{3}}
\end{aligned}
$$

356 where $n$ is the total number of genes and $m$ is the total number of LC states. We then

357 discard all the LC states that are not significant or strongly associated with known

358 technical variations (e.g., cell-specific library complexity and batch information), leading

359 to a lower-dimensional cell-by-LC states matrix $\mathbf{S}$.

360

361 Distance calculation 
362 Distances between cells in $\mathbf{S}$ (the cell-by-LC states matrix) are calculated using cosine

363 distance:

364

$$
K_{a, b}=1-\frac{\sum_{i=1}^{p} S_{a, i} S_{b, i}}{\sqrt{\sum_{i=1}^{p} S_{a, i}^{2}} \sqrt{\sum_{i=1}^{p} S_{b, i}^{2}}}
$$

365

366

367

368

369

370

371

\section{Distance measure in the PC space}

373 The cell-gene relative expression matrix was decomposed by PCA and the significant

374 components were determined using the Tracy-Widom test. Distance between cells was

375 measured by the correlation distance of significant components.

\section{The optimal number of clusters and informative cellular states}

378 We rank the candidate clustering solutions (with different number of clusters) by the

379 silhouette score [11] measured in the PC space. With 2 or more clusters, the silhouette

380 measures the similarity of an individual to its cluster, as compared to other clusters. For

381 each cell, let $d_{b}$ be the lowest dissimilarity to any other cluster and let $d_{w}$ be the

382 average dissimilarity to other cells in its cluster. We calculate the silhouette as 


$$
\text { Silhouette }=\frac{d_{b}-d_{w}}{\max \left(d_{b}, d_{w}\right)}
$$

384 We assign a silhouette score of zero for the default solution of one cluster. An end-user

385 may evaluate the top candidate solutions to determine the optimal number of solutions

386 or specify it with a priori biological knowledge.

388 With the selected number of clusters, we retain LC states that show significant

389 difference among candidate clusters, update the distance matrix and derive the final

390 clustering solution.

\section{Fast processing of large numbers of cells}

393 We classify unknown cells $\mathbf{X}^{\prime}$ efficiently by projecting them into space spanned by the

394 informative LC states learned from a representative sample:

$$
\mathbf{S}^{\prime}=\boldsymbol{\Lambda}_{l}^{-1} \mathbf{G}_{l}^{T} \mathbf{X}^{\prime}
$$

396 where $\Lambda_{l}$ is calculated from the original $\Lambda$ by removing those rows and columns that are

397 not associated with the informative LC states, and $\mathbf{G}_{l}$ is calculated from $\mathbf{G}$ by removing

398 those columns that are not associated with the informative LC states. We can then

399 calculate the cosine similarity between $\mathbf{S}^{\prime}$ and an "average" cell from each cluster and

400 find the cluster with the maximum similarity for each unknown cell.

401

402 Simulation of different cell types 
403 We simulated different cell types from a relatively homogeneous population of cells. We

404 started from the scRNA-seq dataset for sorted CD44 ${ }^{\text {high }}$ Rh41 cells. The data

405 represented 6757 cells with 20,709 genes detected, with $86 \%$ of the entries in the data

406 matrix being zeroes. We considered two factors that have substantial effects on the

407 performance of clustering analysis: the sample size and the degree of difference

408 between cell types.

409

410 We tested five different sample sizes (the total number of cells analyzed): 250,500 ,

4111000,2000 , and 4000 cells. We sampled the selected number of cells from the original

412 set of cells then randomly partitioned them into three sets of variable size, ranging

413 between $10 \%$ and $50 \%$ of the total number of cells.

415 To test the effect of the degree of difference between cell types, we varied the number

416 of DEGs and the simulated difference in gene expression. We chose three sets of

417 genes such that the average expression levels for each set were $g_{1}, g_{2}$, and $g_{3}$, with

$418 \frac{g_{i+1}}{g_{i}}=2, i=1,2$. We then rotated the labels of the three sets of genes to make three

419 different sets of cells with perturbed expression: $g_{1}, g_{2}, g_{3} ; g_{2}, g_{3}, g_{1} ;$ and $g_{3}, g_{1}, g_{2}$. We

420 tested two different numbers of DEGs: 300 and 1200. The simulation setting is

421 summarized in Supplemental Table 4. For each set of parameters, we generated 100

422 random samples for testing. 
425 We evaluate the performance of clustering against true labels of cells by using

426 normalized mutual information (NMI) [14] and variation of information (VI) [13]. We use

427 the R package igraph to calculate both metrics [28].

428

429 Benchmarking

430 We used SC3 (Version 1.4.2), Seurat (Version 2.0.0), Monocle2 (Version 2.4.0) and

431 SIMLR (Version 1.2.1) for comparisons with LCA. We used the default settings for all

432 the packages tested. For SIMLR, when the size of the sample was 1000 or larger, we 433 used SIMLR_Large_Scale() instead of SIMLR().

\section{Rh41 single-cell dataset}

436 The human alveolar rhabdomyosarcoma cell line Rh41 was grown in culture in a 5\%

$437 \mathrm{CO}_{2}$ incubator in $75-\mathrm{cm}^{2}$ vented flasks containing DMEM medium supplemented with

$43810 \%$ FBS and $2 \times$ glutamine until the cells reached $75 \%$ confluence at approximately

$4393.6 \times 10^{6}$ cells. The cells were detached from the flask with $7 \mathrm{~mL}$ of $1 \times$ citrate saline to

440 which $7 \mathrm{~mL}$ of DPBS was added. The cell suspension was then centrifuged at $300 \times g$

441 for $7 \mathrm{~min}$, and the cell pellet was resuspended in $300 \mu \mathrm{L}$ of blocking buffer (rat IgG/PBS)

442 and incubated on ice for $30 \mathrm{~min}$. An aliquot of $50 \mu \mathrm{L}$ of the cells in blocking buffer was

443 transferred to a separate tube for the isotype control. The cells were washed with $1 \mathrm{~mL}$

444 of staining buffer (5\% BSA/PBS) and centrifuged at $300 \times g$ for $5 \mathrm{~min}$. The pellet, which

445 contained approximately $3 \times 10^{6}$ cells, was then incubated with rat IgG2B anti-CD44-

446 Alexa Fluor 488 antibody (R\&D Systems) in staining buffer (15 $\mu \mathrm{L}$ antibody $+135 \mu \mathrm{L}$ of 
447 staining buffer) on ice for $30 \mathrm{~min}$. For the isotype control, approximately 600,000 cells

448 were incubated with $5 \mu \mathrm{L}$ of rat IgG2B-Alexa Fluor 488 (R\&D Systems, Minneapolis,

$449 \mathrm{MN})+45 \mu \mathrm{L}$ of staining buffer on ice for $30 \mathrm{~min}$. After the incubation, both sets of cells

450 were collected by centrifugation, washed with $1 \mathrm{~mL}$ of staining buffer as described

451 above, and resuspended in staining buffer. Flow cytometric analysis was then used to

452 identify the fractions corresponding to the CD44 high and CD44 low populations.

454 For the single-cell experiment, Rh41 cells were grown in culture, harvested, and 455 washed in DPBS as described above. They were then resuspended in PBS/0.2\% BSA 456 at a concentration of $1 \times 10^{6} \mathrm{cells} / \mathrm{mL}$. The $10 \times$ Genomics Single Cell platform performs

457 3' gene expression profiling by poly-A selection of mRNA within a single cell, which uses

458 a cell barcode and UMIs for each transcript. Single-cell suspensions were loaded onto

459 the Chromium Controller according to their respective cell counts to generate

460 approximately 6000 partitioned single-cell GEMs (Gel Bead-in-EMulsions). The library

461 was prepared using the Chromium Single Cell 3' v2 Library and Gel Bead Kit (10x

462 Genomics) in accordance with the manufacturer's protocol. The cDNA content of each

463 sample after cDNA amplification for 12 cycles was quantified, and the quality was

464 checked by High-Sensitivity DNA chip analysis on an Agilent 2100 Bioanalyzer (Agilent

465 Technologies, Santa Clara, CA) at a dilution of 1:6. This quantification was used to

466 determine the final library amplification cycles in the protocol, which were calculated out

467 to 12 cycles. After library quantification and a quality check by DNA 1000 chip (Agilent

468 Technologies), samples were diluted to $3.5 \mathrm{nM}$ for loading onto the HiSeq 4000

469 sequencer (Illumina) with a $2 \times 75$-bp paired-end kit, using the following read length: 26 
470 bp Read1 (10x cell barcode and UMI), 8 bp i7 Index (sample index), and 98 bp Read2

471 (insert). In total, 518 million, 237 million, and 154 million reads were obtained for

472 unsorted, CD44 ${ }^{\text {low }}$, and CD44 ${ }^{\text {high }}$ populations, respectively. The Cell Ranger 2.0.1

473 Single-Cell Software Suite (10x Genomics) was implemented to process the raw

474 sequencing data from the Illumina HiSeq run. This pipeline performed de-multiplexing,

475 alignment (GRCh38/STAR), and barcode processing to generate gene-cell matrices

476 used for downstream analysis.

477

478 After matrix generation, the ribosomal and mitochondria-related genes were filtered out.

480 Rh41 bulk RNA-seq dataset

481 RNA was isolated from the sorted subpopulations by using Trizol (Thermo Fisher

482 Scientific) in accordance with the manufacture's recommendations. RNA libraries were 483 prepared with the KAPA RNA HyperPrep Kit with RiboErase (Roche), using the

484 recommended conditions. Briefly, $200 \mathrm{ng}$ of total RNA was used as input for 485 fragmentation, reverse transcription, and second-strand synthesis. After clean-up, end 486 repair, and A-tailing, NEXTflex adapters (Bioo Scientific, Austin, TX) were ligated to the 487 fragments, and this was followed by 12 cycles of PCR amplification on a C1000 488 Thermal Cycler (Bio-Rad). Paired-end sequencing was performed (151 bases per read) 489 on a HiSeq 4000 system (Illumina). Three replicates were generated. HTSeq [29] was 490 used to produce the count data, and edgeR [30] was used for the DE analysis with TMM 491 normalization. Each replicate was coded as a pair of CD44 ${ }^{\text {high }}$ and CD44 ${ }^{\text {low }}$ in the 492 analysis. 


\section{Statistical analysis}

495 If not specifically stated, the statistical significance was evaluated by the Wilcoxon rank

496 sum test.

497

\section{Abbreviations}

499 CD: Cluster of differentiation DEG: Differentially expressed genes ERMS: fusion-

500 negative embryonal rhabdomyosarcoma FP-ARMS: fusion-positive alveolar

501 rhabdomyosarcoma LCA: Latent cellular analysis NMI: Normalized mutual information

502 PBMC: Peripheral blood mononuclear cell PCA: Principal component analysis t-SNE: t-

503 Distributed stochastic neighbor embedding UMI: Unique molecular identifier VI:

504 Variation of Information

505

\section{Declarations}

507 Acknowledgements

508 We thank Keith A. Laycock for editing the manuscript.

509

$510 \quad$ Funding

511 This study was supported in part by the National Cancer Institute of the National

512 Institutes of Health under Award Number P30CA021765 and by ALSAC.

\section{Availability of data and materials}


The Rh41 scRNA-seq dataset generated during the current study is available from the corresponding author upon reasonable request. The bulk RNA-seq data for sorted CD44 $4^{\text {high }}$ and CD44 ${ }^{\text {low }}$ subpopulations have been deposited in the European

518 Bioinformatics Institute (EMBL-EBI) under accession number EGAS00001002868. The 519 functions used for the data analysis are included in the single_cell_LCA package, which 520 can be installed from Bitbucket (https://bitbucket.org/changde cheng/single cell lca).

\section{References}

523 1. Tang F, Barbacioru C, Wang Y, Nordman E, Lee C, Xu N, Wang X, Bodeau J, Tuch BB, Siddiqui A, et al: mRNA-Seq whole-transcriptome analysis of a single cell. Nat Methods 2009, 6:377-382.

2. Shapiro E, Biezuner T, Linnarsson S: Single-cell sequencing-based technologies will revolutionize whole-organism science. Nat Rev Genet 2013, 14:618-630.

3. Stegle O, Teichmann SA, Marioni JC: Computational and analytical challenges in single-cell transcriptomics. Nat Rev Genet 2015, 16:133-145.

4. Macosko EZ, Basu A, Satija R, Nemesh J, Shekhar K, Goldman M, Tirosh I, Bialas AR, Kamitaki N, Martersteck EM, et al: Highly parallel genome-wide expression profiling of individual cells using nanoliter droplets. Cell 2015, 161:1202-1214.

5. Trapnell C, Cacchiarelli D, Grimsby J, Pokharel P, Li S, Morse M, Lennon NJ, Livak KJ, Mikkelsen TS, Rinn JL: The dynamics and regulators of cell fate decisions are revealed by pseudotemporal ordering of single cells. Nat Biotechnol 2014, 32:381-386.

6. Wang B, Zhu J, Pierson E, Ramazzotti D, Batzoglou S: Visualization and analysis of single-cell RNA-seq data by kernel-based similarity learning. Nat Methods 2017, 14:414-416.

7. Kiselev VY, Kirschner K, Schaub MT, Andrews T, Yiu A, Chandra T, Natarajan KN, Reik W, Barahona M, Green AR, Hemberg M: SC3: consensus clustering of single-cell RNA-seq data. Nat Methods 2017, 14:483-486.

8. Wagner A, Regev A, Yosef N: Revealing the vectors of cellular identity with single-cell genomics. Nat Biotechnol 2016, 34:1145-1160.

9. Hicks SC, Townes FW, Teng M, Irizarry RA: Missing data and technical variability in single-cell RNA-sequencing experiments. Biostatistics 2017.

10. Patterson N, Price AL, Reich D: Population structure and eigenanalysis. PLoS Genet 2006, 2:e190. 
11. Rousseeuw PJ: Silhouettes - a graphical aid to the interpretation and validation of cluster-analysis. Journal of Computational and Applied Mathematics 1987, 20:53-65.

12. Tibshirani $R$, Walther $\mathrm{G}$, Hastie $\mathrm{T}$ : Estimating the number of clusters in a data set via the gap statistic. Journal of the Royal Statistical Society Series BStatistical Methodology 2001, 63:411-423.

13. Meilă M: Comparing clusterings-an information based distance. Journal of Multivariate Analysis 2007, 98:873-895.

14. Danon L, Diaz-Guilera A, Duch J, Arenas A: Comparing community structure identification. Journal of Statistical Mechanics-Theory and Experiment 2005.

15. Zheng GX, Terry JM, Belgrader P, Ryvkin P, Bent ZW, Wilson R, Ziraldo SB, Wheeler TD, McDermott GP, Zhu J, et al: Massively parallel digital transcriptional profiling of single cells. Nat Commun 2017, 8:14049.

16. Tirosh I, Izar B, Prakadan SM, Wadsworth MH, 2nd, Treacy D, Trombetta JJ, Rotem A, Rodman C, Lian C, Murphy G, et al: Dissecting the multicellular ecosystem of metastatic melanoma by single-cell RNA-seq. Science 2016, 352:189-196.

17. Dong C, Martinez GJ: T cells: the usual subsets (poster). 2010.

18. Chen X, Stewart E, Shelat AA, Qu C, Bahrami A, Hatley M, Wu G, Bradley C, McEvoy J, Pappo A, et al: Targeting oxidative stress in embryonal rhabdomyosarcoma. Cancer Cell 2013, 24:710-724.

19. Li F, Tiede B, Massague J, Kang Y: Beyond tumorigenesis: cancer stem cells in metastasis. Cell Res 2007, 17:3-14.

20. Yan Y, Zuo X, Wei D: Concise Review: Emerging Role of CD44 in Cancer Stem Cells: A Promising Biomarker and Therapeutic Target. Stem Cells Transl Med 2015, 4:1033-1043.

21. Rudzinski ER, Anderson JR, Lyden ER, Bridge JA, Barr FG, Gastier-Foster JM, Bachmeyer K, Skapek SX, Hawkins DS, Teot LA, Parham DM: Myogenin, AP2beta, NOS-1, and HMGA2 are surrogate markers of fusion status in rhabdomyosarcoma: a report from the soft tissue sarcoma committee of the children's oncology group. Am J Surg Pathol 2014, 38:654-659.

22. Dumais ST: Latent semantic analysis. Annual Review of Information Science and Technology 2004, 38:189-230.

23. Pearson K: On lines and planes of closest fit to systems of points in space. The London, Edinburgh, and Dublin Philosophical Magazine and Journal of Science 1901, 2:559-572.

24. Hotelling $\mathrm{H}$ : Analysis of a complex of statistical variables into principal components. Journal of Educational Psychology 1933, 24:417-441.

25. Tracy $\mathrm{CA}$, Widom $\mathrm{H}$ : Level-spacing distributions and the Airy kernel. Physics Letters B 1993, 305:115-118.

26. Johnstone IM: On the distribution of the largest eigenvalue in principal components analysis. Annals of Statistics 2001, 29:295-327.

27. $\mathrm{Ng} A Y$, Jordan MI, Weiss Y: On spectral clustering: Analysis and an algorithm. In Advances in neural information processing systems; 2002. 2002: 849-856. 
596 28. Csardi G, Nepusz T: The igraph software package for complex network

597 research. InterJournal 2006, Complex Systems:1695.

598 29. Anders S, Pyl PT, Huber W: HTSeq--a Python framework to work with highthroughput sequencing data. Bioinformatics 2015, 31:166-169.

600 30. Robinson MD, McCarthy DJ, Smyth GK: edgeR: a Bioconductor package for differential expression analysis of digital gene expression data.

603 Bioinformatics 2010, 26:139-140.

604 $S$, Jenkins SL, Jagodnik KM, Lachmann A, et al: Enrichr: a comprehensive gene set enrichment analysis web server 2016 update. Nucleic Acids Res 2016, 44:W90-97. 


\section{Figure Legends}

610

611 Figure 1. Overview of the workflow of LCA. LCA infers LC states from full expression

612 matrices. Explicit gene filtering is not necessary. LCA converts the raw transcript count

613 data to gene fractions and then performs log transformations. The algorithm features a

614 dual-space model search, generating candidate clustering models based on the cosine

615 similarity matrix in the LC space. Candidate models are then ranked based on the

616 silhouettes measured in the PC space.

617

618 Figure 2. Benchmarking of LCA against four other methods with simulated datasets. (A)

619 The number of clusters inferred by LCA, SC3, and Seurat was compared for seven

620 simulated datasets: the signal strength increases from simulated datasets 1 to 6 . Each

621 simulated dataset contained 100 randomly generated samples. (B) Comparison of the

622 clustering performance of LCA, SC3, Seurat, Monocle2, and SIMLR, as measured by

623 variation of information, across the seven simulated datasets. For LCA, SC3, and

624 Seurat, the number of clusters was inferred during the analysis. For Monocle2 and

625 SIMLR, we used the correct number of clusters. (C) Comparison of the clustering

626 performance as measured by normalized mutual information. (D) Clustering

627 performance scalability of LCA and SC3. Each panel represents the results obtained

628 with a different percentage of cells used for training (from $5 \%$ to $25 \%$ ). The total number

629 of cells in the sample was 4000 . The signal in this comparison was relatively weak. (E)

630 Comparison of the clustering performance scalability of LCA and SC3 when the signal

631 was strong. (F) Comparison of the running times of LCA and SC3. 
633 Figure 3. Investigation of PBMC heterogeneity by using LCA. We applied LCA to data

634 from Zheng et al. ${ }^{25}$ for 55,000 cells representing a combination of six sorted T-cell

635 populations. LCA identified five cell populations with biologically meaningful LC states.

636 (A) Cell distance matrix, sorted by source ID. (B). Cell distance matrix, sorted by LCA

637 clustering results. (C) The first LC state captured the difference between the naïve and

638 differentiated T cells, whereas the second LC state captured the difference between the

639 CD4+ and CD8+ cells. (D) LCA further revealed heterogeneity in the CD4+ helper T

640 cells and CD8+ cytotoxic T cells.

642 Figure 4. Reanalysis of melanoma cellular data [16] with LCA. We applied LCA to 1169

643 malignant cells from eight tumors and to 2588 stromal cells. LCA identified 18 clusters

644 with a clear biological interpretation. LCA further revealed subtle diversity among the

645 infiltrating T cells. (A) t-SNE plot of the clustering results for malignant cells, contrasting

646 the LCA results and original results. (B) t-SNE plot of the clustering results for stromal

647 cells, contrasting the LCA results and original results. (C) Enrichment of gene markers

648 in T-cell clusters.

649

650 Figure 5. LCA analysis of Rh41 cells, correcting for batch effects. (A) t-SNE plots of

651 clustering results, colored according to source ID or cluster ID. (B) Cellular expression

652 patterns of DEGs identified in bulk RNA-seq. (C) Violin plot of expression patterns of

653 DEGs from bulk RNA-seq in cells classified by source and cluster ID. 
654 Figure 1. Overview of the workflow of LCA.

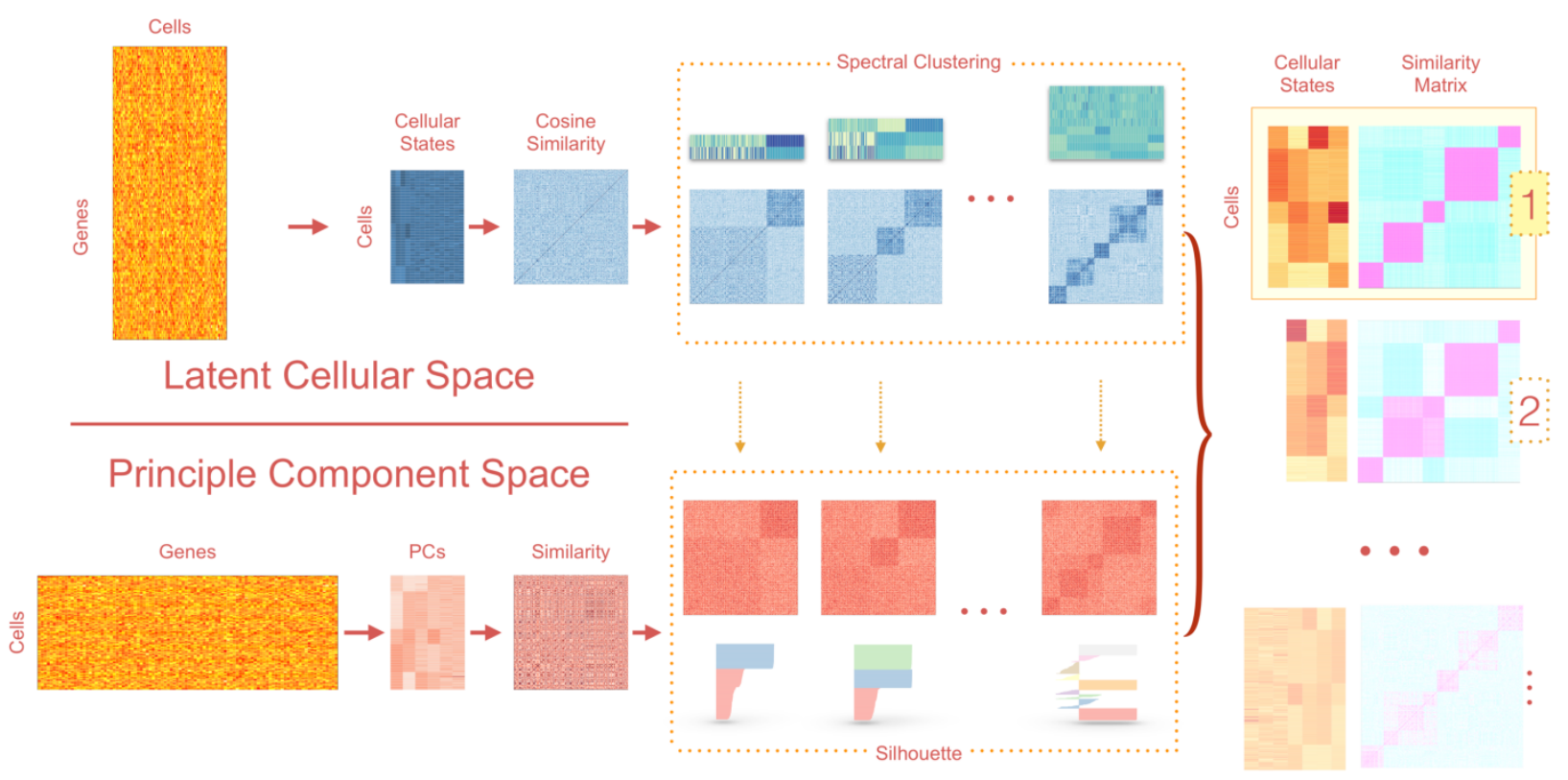


Figure 2. Benchmarking of LCA against four other methods with simulated datasets.
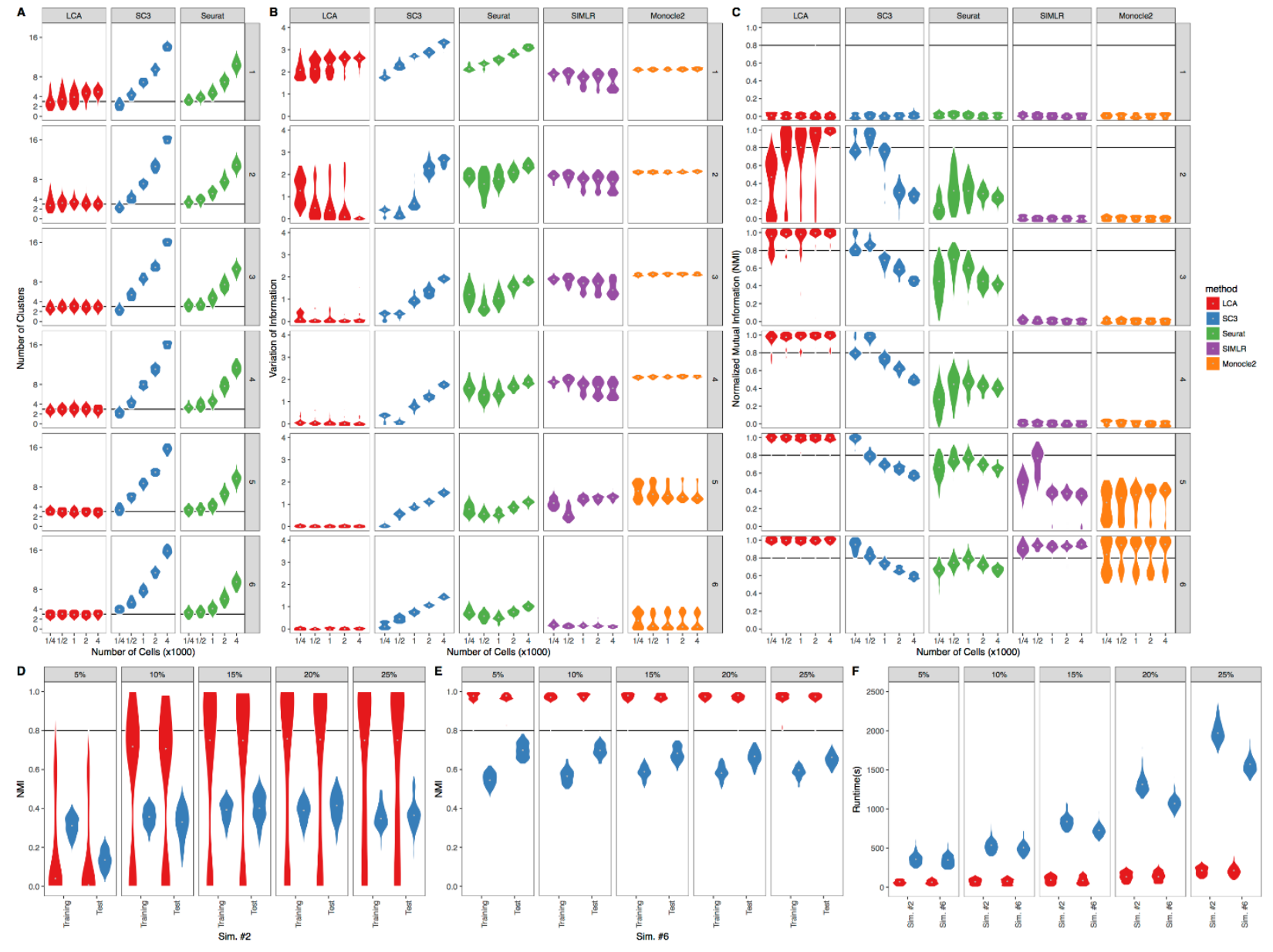
660 Figure 3. Investigation of PBMC heterogeneity by using LCA.

A

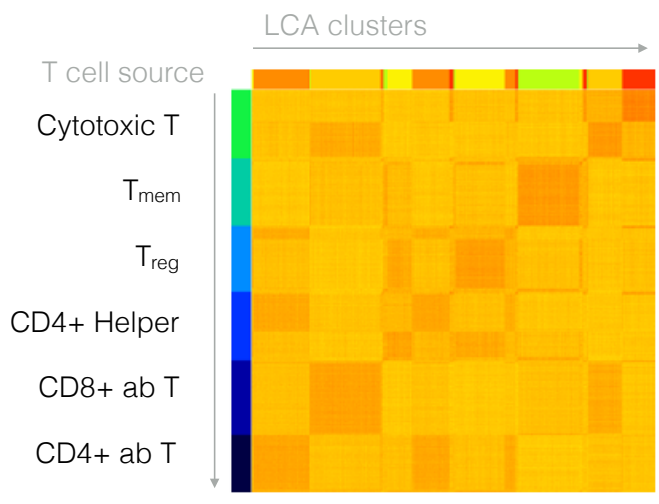

C
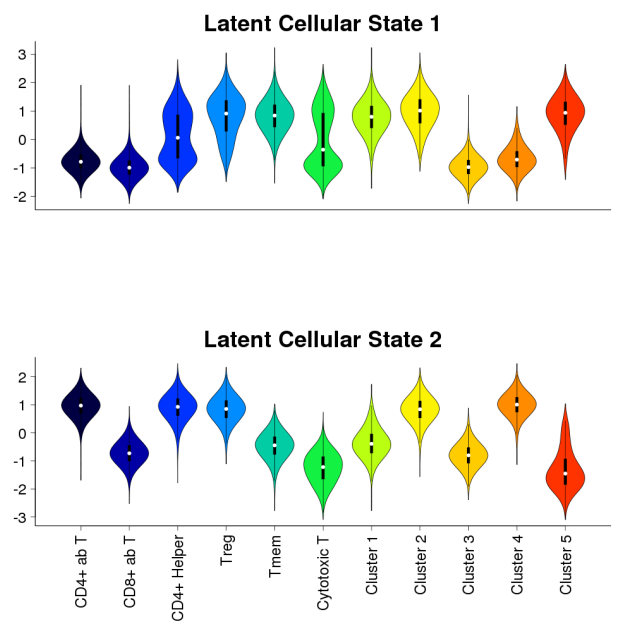

B

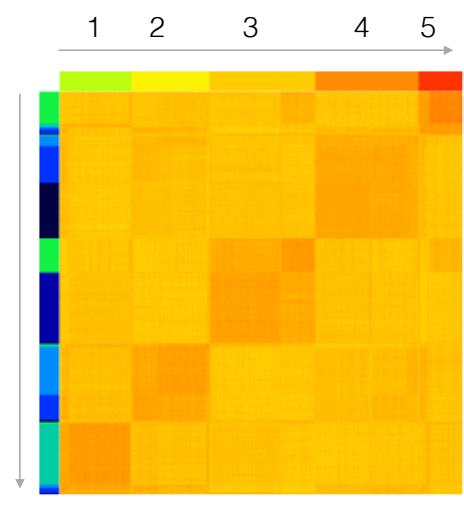

D

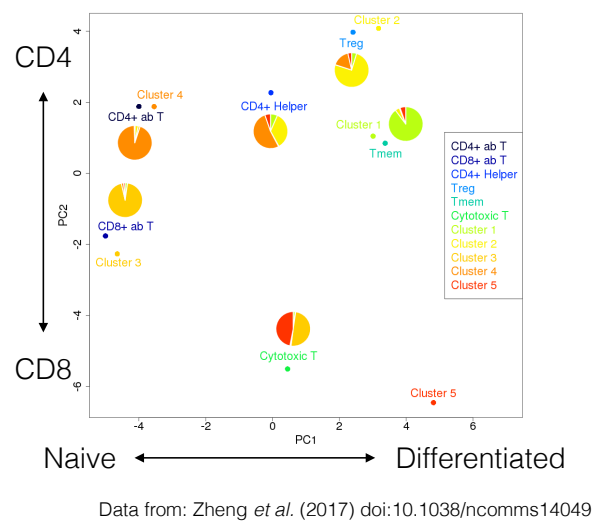

661

662 


\section{Figure 4. Reanalysis of melanoma cellular data with LCA.}
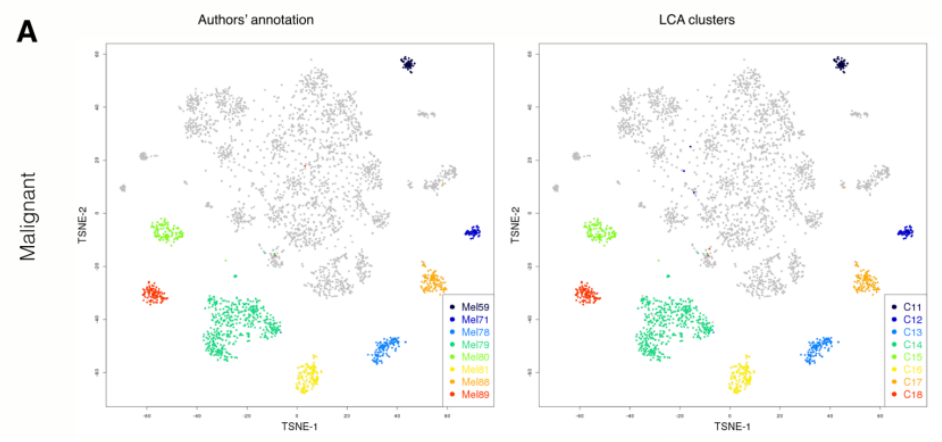

B
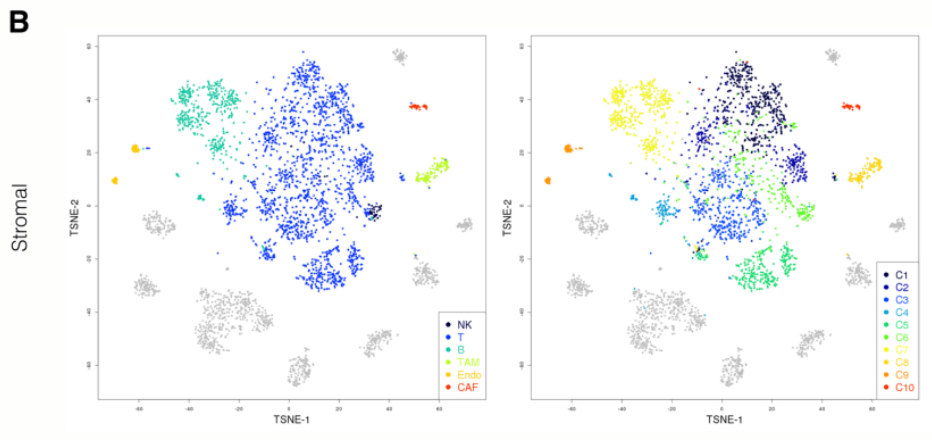

C

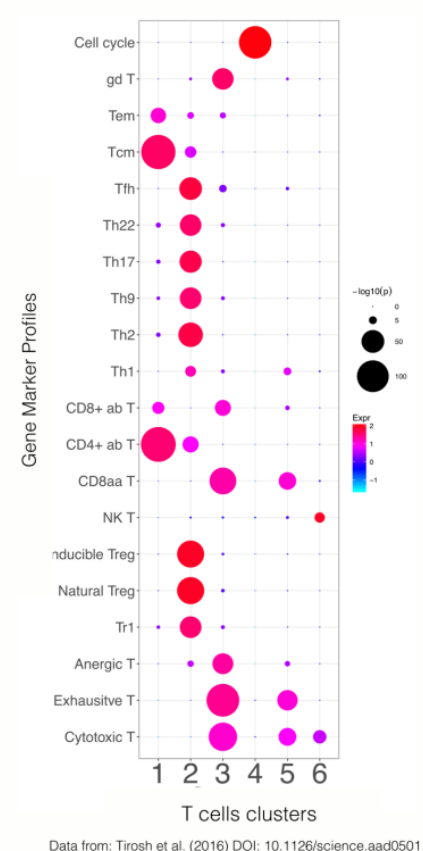

665 
666 Figure 5. LCA analysis of Rh41 cells, correcting for batch effects.

A

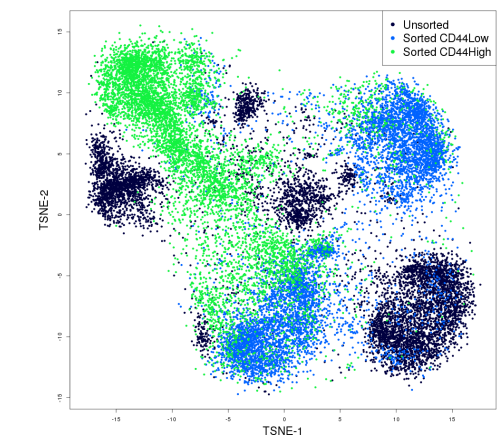

B

Over-expressed genes in bulk CD44 high 294 genes

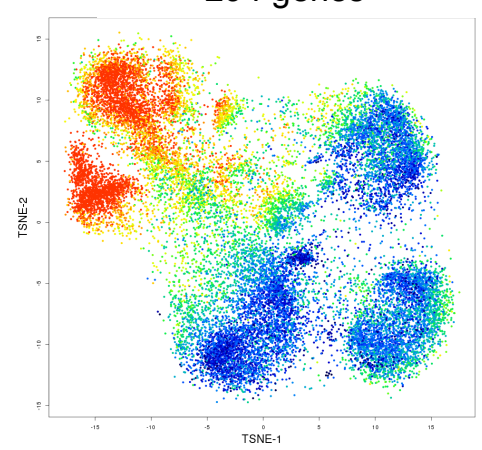

By Cluster

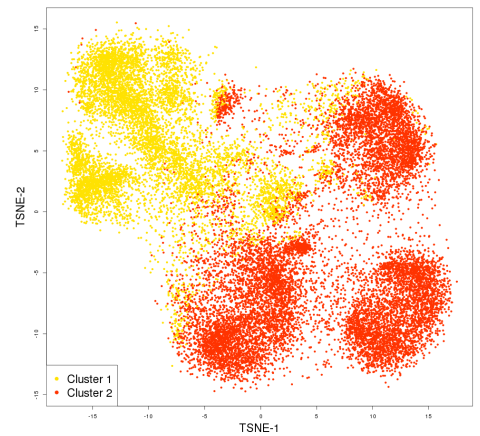

Over-expressed genes in bulk CD44 low 62 genes

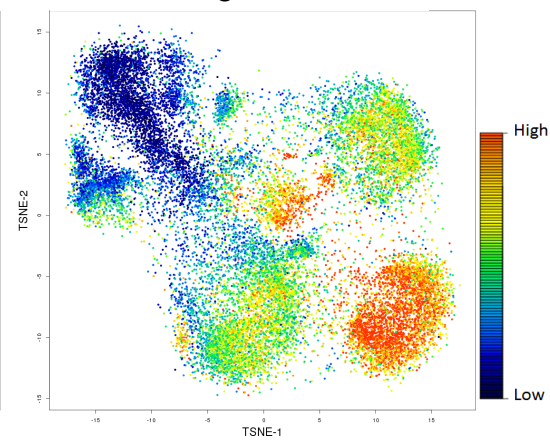

C

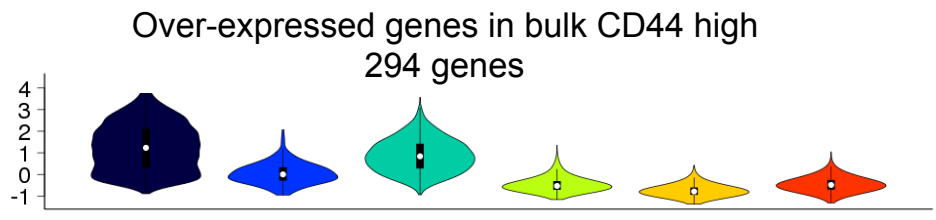

Over-expressed genes in bulk CD44 low

62 genes

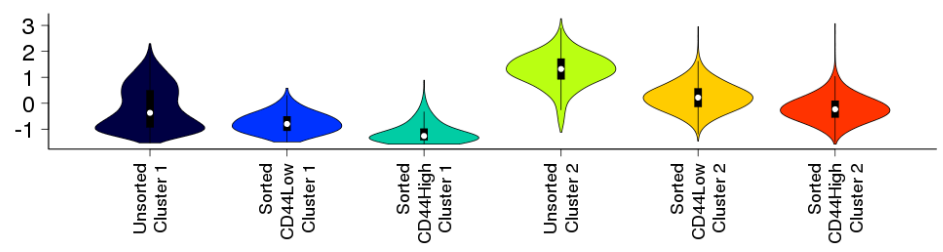


668 Supplemental Figure 1. Single-cell analysis of Rh41 cells with strong batch effects. (A)

669 Distance matrix visualized as a heat map. (B) Flow cytometry confirmed a bimodal

670 expression pattern of CD44 in Rh41 cells. (C) The first two PCs for the bulk RNA-seq

671 data showed strong batch effects. (D) t-SNE plot of cells, including sorted and unsorted

672 samples, colored according to source/cluster ID. (E) The first LC state was significantly

673 associated with batch information.

674 
675 Supplemental Figure 1. Single-cell analysis of Rh41 cells with strong batch effects.

A

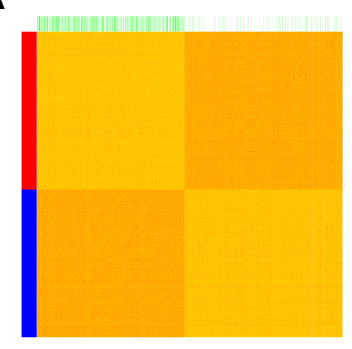

B

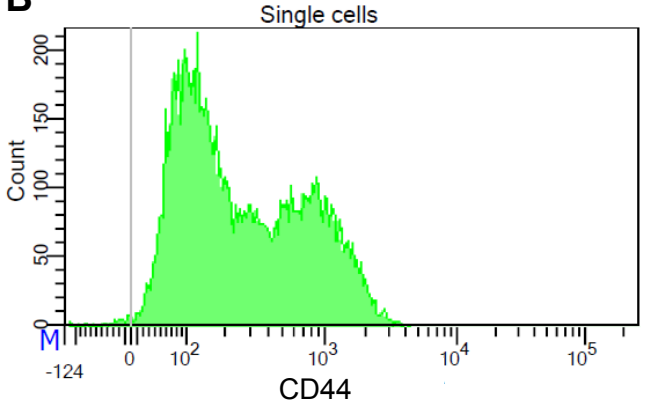

By Source

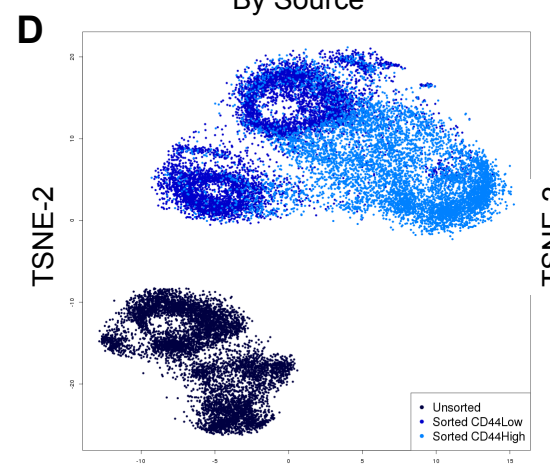

TSNE-1

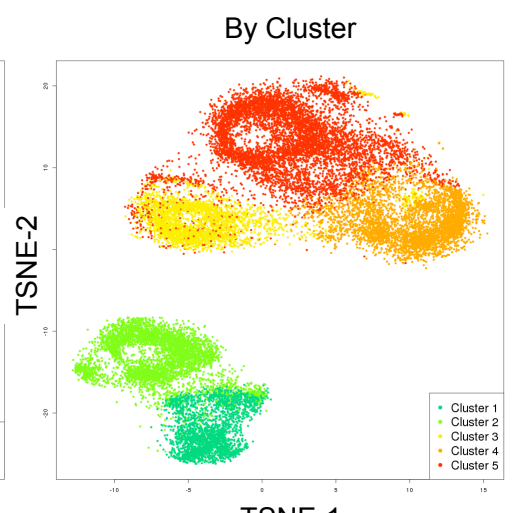

TSNE-1

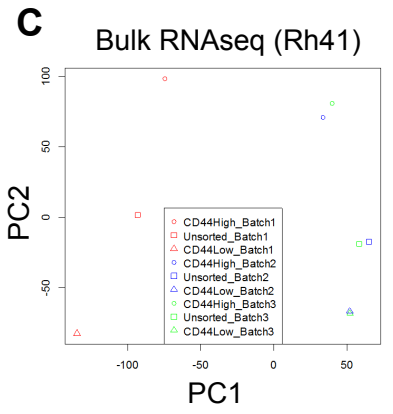

E Latent Cellular State 1

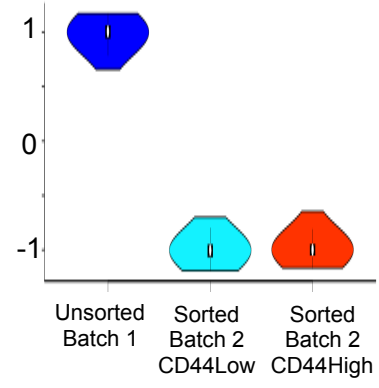


678 Supplementary Table 1. The results of applying LCA and SC3 to the dataset of Tirosh

679 et al. ${ }^{26}$, using normalized mutual information.

\begin{tabular}{|l|c|c|}
\hline & LCA & SC3 \\
\hline All cells & 0.77 & 0.69 \\
\hline Malignant cells & 0.99 & 0.99 \\
\hline All stromal cells & 0.53 & 0.42 \\
\hline All cells excluding T cells & 0.97 & 0.92 \\
\hline Stromal excluding T cells & 0.90 & 0.71 \\
\hline
\end{tabular}

Supplementary Table 2. Expression profile of differential expressed genes (in a standalone excel file).

689 Supplementary Table 3. ChEA analysis by Enrichr[31] of 361 overexpressed genes in 690 the CD44 ${ }^{\text {low }}$ subpopulation of Rh41 (in a standalone excel file). 
693 Supplementary Table 4. Summary of simulated datasets.

\begin{tabular}{|c|c|c|c|c|}
\hline Signal & Number of & Mean Gene & Mean Gene & Mean Gene \\
Scale & Genes & Fraction in Group 1 & Fraction in Group 2 & Fraction in Group 3 \\
\hline Perturbed & & & & \\
\hline Sim. \#1 & 300 & $1 \mathrm{e}-5$ & $2 \mathrm{e}-5$ & $4 \mathrm{e}-5$ \\
\hline Sim. \#2 & 300 & $2 \mathrm{e}-5$ & $4 \mathrm{e}-5$ & $8 \mathrm{e}-5$ \\
\hline Sim. \#3 & 300 & $4 \mathrm{e}-5$ & $8 \mathrm{e}-5$ & $1.6 \mathrm{e}-4$ \\
\hline Sim. \#4 & 1200 & $1 \mathrm{e}-5$ & $2 \mathrm{e}-5$ & $4 \mathrm{e}-5$ \\
\hline Sim. \#5 & 1200 & $2 \mathrm{e}-5$ & $4 \mathrm{e}-5$ & $8 \mathrm{e}-5$ \\
\hline Sim. \#6 & 1200 & $4 \mathrm{e}-5$ & $8 \mathrm{e}-5$ & $1.6 \mathrm{e}-4$ \\
\hline
\end{tabular}

694

695 